\title{
Quantitative trait loci-dependent analysis of a gene co-expression network associated with Fusarium head blight resistance in bread wheat (Triticum aestivum L.)
}

\author{
Karl G Kugler ${ }^{1 \dagger}$, Gerald Siegwart ${ }^{2 \dagger}$, Thomas Nussbaumer ${ }^{1}$, Christian Ametz ${ }^{2}$, Manuel Spannagl ${ }^{1}$, Barbara Steiner ${ }^{2}$, \\ Marc Lemmens ${ }^{2}$, Klaus FX Mayer ${ }^{1}$, Hermann Buerstmayr ${ }^{2}$ and Wolfgang Schweiger ${ }^{2 *}$
}

\begin{abstract}
Background: Fusarium head blight (FHB) caused by Fusarium graminearum Schwabe is one of the most prevalent diseases of wheat (Triticum aestivum L.) and other small grain cereals. Resistance against the fungus is quantitative and more than 100 quantitative trait loci (QTL) have been described. Two well-validated and highly reproducible QTL, Fhb1 and Qfhs.ifa-5A have been widely investigated, but to date the underlying genes have not been identified.

Results: We have investigated a gene co-expression network activated in response to $F$. graminearum using RNA-seq data from near-isogenic lines, harboring either the resistant or the susceptible allele for Fhb1 and Qfhs.ifa-5A. The network identified pathogen-responsive modules, which were enriched for differentially expressed genes between genotypes or different time points after inoculation with the pathogen. Central gene analysis identified transcripts associated with either QTL within the network. Moreover, we present a detailed gene expression analysis of four gene families (glucanases, NBS-LRR, WRKY transcription factors and UDP-glycosyltransferases), which take prominent roles in the pathogen response.

Conclusions: A combination of a network-driven approach and differential gene expression analysis identified genes and pathways associated with Fhb1 and Qfhs.ifa-5A. We find G-protein coupled receptor kinases and biosynthesis genes for jasmonate and ethylene earlier induced for Fhb1. Similarly, we find genes involved in the biosynthesis and metabolism of riboflavin more abundant for Qfhs.ifa-5A.
\end{abstract}

Keywords: Triticum aestivum, Bread wheat, Fusarium graminearum, Fusarium head blight, Fhb1, Qfhs.ifa-5A, Transcriptome, Gene co-expression network, RNA-seq

\section{Background}

Bread wheat (Triticum aestivum L.) is one of the most important food crops worldwide. $20 \%$ of the human calorie and protein uptake derive from wheat. One of its most prevalent and destructive pathogens is the fungus Fusarium graminearum (teleomorph Gibberella zeae, Schwabe) $[1,2]$. F. graminearum frequently infects wheat and other small grain cereals in temperate regions throughout the

\footnotetext{
* Correspondence: wolfgang.schweiger@boku.ac.at

${ }^{\dagger}$ Equal contributors

${ }^{2}$ Institute for Biotechnology in Plant Production, IFA-Tulln, University of

Natural Resources and Life Sciences, A-3430 Tulln, Austria

Full list of author information is available at the end of the article
}

world. Especially under humid weather conditions spores that have overwintered in remaining plant debris on the field reach the flowering wheat head via splash water, from where the germinating fungus penetrates the more susceptible floral tissue. The resulting disease Fusarium head blight (FHB) annually accounts for severe losses in grain yield and also quality due to the contamination with mycotoxins produced by the fungus. Among these deoxynivalenol (DON) holds a key position. DON is a potent inhibitor of protein biosynthesis and constitutes a serious threat to human and animal health in food and feed [3]. This has, among others, prompted the European Union to enact maximum tolerated levels in food [4] and 
advisory levels were issued by the Food and Drug Administration in the United States. Consequently, developing high yielding and $F$. graminearum resistant varieties is of high priority for breeders. Despite its economic relevance, the genomic sequence of wheat is not yet available due to its sheer size $(\sim 17 \mathrm{~Gb})$ and its highly repetitive nature $[5,6]$. However, a large body of publications aiming towards genetic mapping of resistance genes against $F$. graminearum has been published in the last 14 years and so far over 100 quantitative trait loci (QTL) have been described to contribute to resistance [7]. Two highly reproducible and large-effect QTL are Fhb1 located on the short arm of chromosome 3B [8] and Qfhs.ifa-5A on chromosome 5A [9]. Depending on the genetic background both reduce disease symptoms by $20-25 \%$ and confer either type II resistance against spreading of the disease $(F h b 1)$ or type I resistance against initial penetration (Qfhs.ifa-5A) $[9,10]$. Fhb1 was linked to the higher ability to enzymatically inactivate DON by glycosylation [11], but recent reports also associate the higher formation of phenylpropanoids [12] or a non-responsive susceptibility factor, WFhb1_c1 [13] with the activity of the QTL. No functional evidence has been proposed for Qfhs.ifa-5A. A donor of both QTL is the CIMMYT (http://www.cimmyt.org) derived line CM-82036, a progeny of the prominent resistance source Sumai-3. CM-82036 also encodes for multiple minor effect resistance QTL, which provide the line with a significantly higher level of resistance when compared to a near-isogenic line stacking both $F h b 1$ and Qfhs.ifa-5A resistance alleles in a susceptible background $[10,14]$. Recent years have seen multiple transcriptomic and proteomic studies investigating the F. graminearum/wheatinteraction, which have helped developing an understanding of the general response against the fungus (reviewed in [2]), but these did not lead to the identification of QTLrelated resistance genes so far.

RNA-sequencing technology is well established as an alternative to microarrays. The major obstacle for the analysis of the entire wheat transcriptome is the availability of a suitable mapping reference covering the gene space of the yet unsequenced species. Establishing the gene-space as a reference is even more challenging as the three homeologous genomes of polyploid bread wheat share a high level of sequence similarity. A recent study [15] tried to overcome these limitations by combining short reads from Illumina technology with 454 data in a two-stage assembly. The TriFLDB [16] database collected available full-length coding sequences from wheat over the last years. Currently, efforts are underway to assemble the wheat genome entirely using chromosome arm sorting [17], genotyping by sequencing [18] and whole genome profiling approaches [19]. The most complete assembly of the $T$. aestivum gene space is described by the released wheat low-copy-number genome
(LCG) assembly [5], generated from 454 sequences and reference as well as progenitor genomes, which provides partial sequences of an estimated number of $94-96 \mathrm{k}$ genes. In addition, the transcriptome of the close relative barley (Hordeum vulgare L.) comprising more than $26 \mathrm{k}$ genes has been annotated on a WGS assembly and anchored to the physical map [20]. These data, the wheat LCG assembly and the homology to the complete barley gene space provide a novel and unique reference for RNAprofiling studies, allowing a high specificity and coverage of the transcriptome.

To gain novel insights into the defense response of wheat against $F$. graminearum using these newly available data resources, we have sequenced the transcriptome of five differently resistant genotypes, comprising a set of four near-isogenic lines (NILs) harboring either, both or none of the resistance alleles of Fhb1 and Qhfs. ifa-5A in the susceptible background of the German spring wheat cultivar Remus and the highly resistant QTL-donor line CM-82036. While most of the existing, microarray-based analyses aimed at analyzing single genes, we here provide a transcriptome-wide approach and focus on investigating the interaction of genes. Several studies have demonstrated the power of co-expression networks for detecting groups of genes that react in a coordinated effort against pathogen response, e.g. in cucumber and rice $[21,22]$. In the present study we introduce a networkdriven approach, which led to the identification of groups of genes that form functional clusters of co-expressed genes. Additionally, we screened for single genes that occupy central positions within the newly established network (e.g. hub genes). We find that these putative key genes in the response to $F$. graminearum are members of prominent pathogenesis-related gene families. We further investigate differential expression patterns observed for the glucanase, nucleotide-binding site leucine-rich repeat (NBS-LRR), WRKY and UDP-glycosyltransferase (UGT) gene families, which hold relevant positions in our analysis.

\section{Results}

\section{Data harvesting, processing and quality control}

We extracted RNA from spike tissue of five different wheat genotypes that were treated with a $F$. graminearum spore suspension or mock 30 and 50 hours after inoculation (hai). All lines showed distinct levels of resistance after point inoculation in green house trials $[10,14]$. The lines comprised a set of four NILs that harbor either of the F. graminearum-resistance QTL Fhb1 (NIL2, moderately resistant) or Qfhs.ifa-5A (NIL3, moderately resistant), both of these QTL (NIL1, resistant) or none of them (NIL4, susceptible) in the genetic background of the F. graminearum susceptible German spring wheat cultivar Remus. These lines are at least 96\% isogenic as shown with DArT 
markers [14], but do contain QTL-unrelated, yet linked genes from the original QTL donor in the introgressed section. Additional samples were collected from the highly resistant QTL-donor line CM-82036, which encodes in addition to $F h b 1$ and Qfhs.ifa-5A for multiple minor-effect QTL. Samples were sequenced on an Illumina HiSeq2000 platform, which summed up to a total of $1,827 \mathrm{~Gb}$ raw sequences (Additional file 1). RNA-Seq reads were compared against public wheat full-length cDNA [16] to ensure the quality and coverage of genes along the entire length (Additional file 2). This allowed to map reads on the LCG assembly [5] resulting in 233,780 Cuffmerge transcripts, out of which 151,853 (65\%) transcripts are expressed in all five genotypes (Table 1). To assess the progress of the disease, reads were compared to the F. graminearum transcriptome [23]. In average $87 \mathrm{k}$ reads ( $0.3 \%$ from the average of total reads) were matching F. graminearum genes for samples inoculated with spore suspensions and no more than about $1.8 \mathrm{k}$ reads in the mock-treated samples (Additional file 3). This observation can be explained by contaminations, mapping errors or conserved domains. One particular mock treated sample (NIL2, 50 hai, replicate 3) contained an unexpected high number of reads (10.7 k reads) that matched F. graminearum genes and was therefore excluded from further analysis. While samples taken at 50 hai showed in general a higher abundance of $F$. graminearum-mapped reads than 30 hai samples, we could not detect significant differences between the infected lines at any time point (Additional file 4).

Cuffdiff [24] was used to extract differentially expressed genes (Benjamini-Hochberg correction [25] (BH); $p<0.1$; see Methods). As the LCG contigs are in general short (average length: $714 \mathrm{bp}$ ) and represent only partial genes due to the high (genic) sequence redundancy in hexaploid wheat, we used the recently published barley high confidence genes $(N=26,159$, [18]) to filter the previously generated fragmented Cufflinks transcripts for gene candidates. Thereby, with the barley best bidirectional hit $(\mathrm{BBH})$, we were able to link 16,964 transcripts to barley genes. Mapping to barley homologs has two major

Table 1 Mapping of RNA-seq data

\begin{tabular}{cccc}
\hline Line & Cufflinks & Barley BBH & DEG (barley BBH) \\
\hline CM-82036 & 183,540 & 15,360 & $1,956(13 \%)$ \\
NIL1 & 189,486 & 15,797 & $1,781(11 \%)$ \\
NIL2 & 196,078 & 15,734 & $2,067(13 \%)$ \\
NIL3 & 192,584 & 15,676 & $1,954(12 \%)$ \\
NIL4 & 186,755 & 15,680 & $2,005(13 \%)$ \\
\hline
\end{tabular}

Overview of generated cufflinks genes with the corresponding number of barley best bi-directional hits (BBH) as well as the number of differentially expressed genes (DEG) is given. Overall 233,780 cufflinks genes were found in all five lines, with 151,853 genes in common. impacts: It drastically reduces the number of analyzed transcripts in the analysis and also wheat-specific genes with no barley homologs may be lost. Moreover, a differentiation between homeologous genes from different genomes is not always possible. However, the remaining transcripts have a higher quality in terms of gene calling confidence. Additionally, for these data trustworthy sets of Gene Ontology (GO) and Interpro annotations exist for analyzing the remaining transcripts. Overall these sets comprised 9,776 genes with GO annotations and 14,164 genes with Interpro annotations. Therefore, we performed the subsequent enrichment analyses and network inference on the barley $\mathrm{BBH}$ reduced subset.

Genotype-specific differentially expressed genes link Fhb1 to early induction of jasmonate and ethylene biosynthesis and Qfhs.ifa-5A to riboflavin biosynthesis and lipid binding

With Cuffcompare $233 \mathrm{k}$ cufflinks genes were combined and tested for differential expression by comparing F. graminearum inoculated samples with the respective mock-treated sample (FDR adjusted $p$-value $<0.1$ ). Per genotype between 183,540 (CM-82036) to 196,078 (NIL2) Cufflinks genes were assembled. BBH assigned barley genes were found for 15,360 (CM-82036) to 15,797 (NIL1) Cufflinks genes. In average $8 \%$ of those genes were differentially expressed, for Cufflinks genes with $\mathrm{BBH}$ linkage $11-13 \%$. To provide a more granular insight into differentially expressed genes, we analyzed common differentially expressed genes (DEG) for 30 hai and 50 hai separately. A list of all analyzed differentially expressed genes is provided in Additional file 5.

The earlier sampling time point 30 hai was characterized by a pronounced response of the resistant CM82036 (Figure 1a). From the 1,302 DEG identified in total, 289 were differentially expressed only for CM-82036 compared to 114 DEG, which were shared between all five genotypes. The group of DEG shared only between NILs 1-4 and also the number of DEG specific for each NIL comprised only few genes (10 to 41 ). 50 hai a large part (618) of the 2,470 DEG was significantly changed for all genotypes. At this time point, in contrast to $30 \mathrm{hai}$, all genotypes exhibited a similarly strong response in terms of DEGs (Figure 1b). Also a large group of DEG was shared between the four NILs (114) representing the response of genes in the genetic background of the susceptible recurrent parent Remus.

GO terms obtained via topGO [26] for these contrasts represented genotype dependent defense responses (Additional file 6 for 30 hai and Additional file 7 for 50 hai). Regardless of QTL all genotypes shared essential pathogenesis associated pathways at 30 hai: These included the biosynthesis of phenylpropanoids and polyamins and also genes involved in the reduction of oxidative stress and chitinases. We also found a glutamate synthase more 


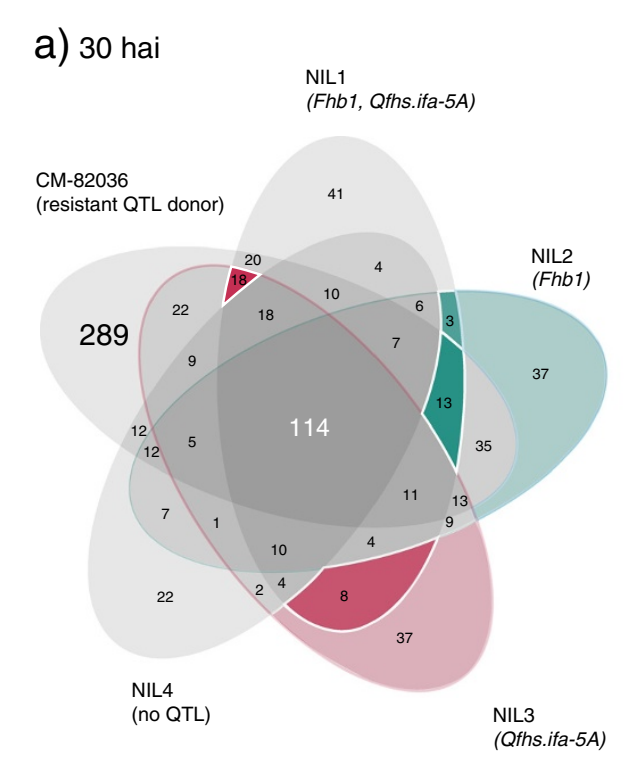

b) 50 hai

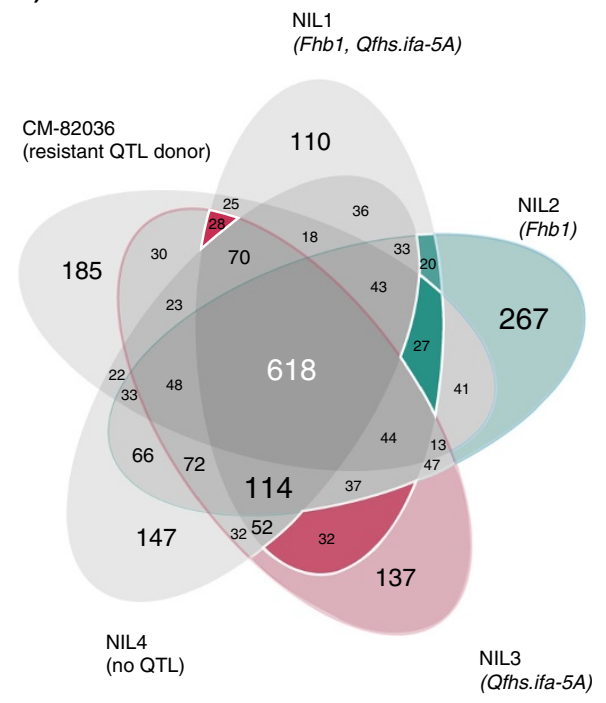
inoculation (hai) (a) and 50 hai (b) for the investigated genotypes (CM-82036, NIL1 (harboring both resistance QTL, Fhb1 and Qfhs.ifa-5A), NIL2 (Fhb1), NIL3 (Qhfs.ifa-5A) and NIL4 (no QTL)) as well as genes shared between them in the respective intersections. Intersections of lines sharing either of the two QTL harbor genes associated with these QTL. These are highlighted in cyan (Fhb1) or magenta (Qfhs.ifa-5A).

abundant 30 hai and an amino acid transporter more abundant at 50 hai. Both may be linked to multiple pathogen-induced reactions in the primary and secondary metabolism [27,28]. Moreover, an UDP-N-acetylmuramate dehydrogenase was also upregulated 30 hai, which potentially acts in biosynthesis of amino sugars used for posttranslational protein modification. 50 hai we observed additional terms related to ubiquitination and the biosynthesis of tryptophan.

The response unique for CM-82036 comprised a high number of terms corresponding to signaling events and transcription factors at the early time point and also terms corresponding to the biosynthesis of trehalose and terpenoids. The response at 50 hai included UGTs, cytochrome P450 monooxygenases (CYP) and terms related to the primary metabolism involved in amino acid biosynthesis and gluconeogenesis.

Genes associated with the activity of Fhb1 or Qfhs.ifa$5 A$ should be represented by DEG shared by NILs harboring these QTL (highlighted sections in Figure 1). In the section shared by NIL2 (resistant allele of Fhb1) and NIL1 (both QTL) and in the section shared by both NILs and CM-82036 we identified 16 genes collectively at 30 hai and 47 at 50 hai. Similarly, 26 and 60 genes were shared in lines harboring the resistance allele of Qfhs.ifa-5A (NIL1 and NIL3 containing Qfhs.ifa-5A only and optionally CM-82036). We also looked at the differentially expressed genes unique for the genotypes harboring only either of both QTL (NIL2 and NIL3), as the activity of QTL-related genes might not be similarly significantly changed at the observed time point in all lines harboring these QTL due to the different resistance levels.

The specific response of the NIL2 containing Fhb1 was characterized by the early upregulation of transcription factors and biosynthesis genes for jasmonic acid (JA) and ethylene (ET). Both signaling molecules regulate defense responses in plants against biotic stresses. At 50 hai we found terms related to translation, protein folding and ribosomal protein more abundant. For transcripts shared between lines with $F h b 1$ we identified GO terms relating to protein secretion and signal transduction (G protein-related) at 30 hai and terms related to the metabolism of glutamine at 50 hai. Lines containing Qfhs.ifa-5A (NIL1 and NIL3) showed higher abundance of gene transcripts related to the tryptophan biosynthesis pathway already at 30 hai and for genes related to lipid binding at 50 hai. GO terms identified in the shared sections are involved in riboflavin production and ET biosynthesis (30 hai). We also found a transcript encoding a glutamate-gated ion channel (30 hai), which controls $\mathrm{Ca}^{2+}$-influx into the cell. Similarly to $F h b 1$, these sections also included terms for ribosome biogenesis and protein translation. 


\section{Gene co-expression network analysis identifies defense-associated modules}

We analyzed the co-expression data from the barleymapped transcripts of all samples to infer a gene coexpression network specific for the observed conditions. In contrast to the detection of single DEG, this approach takes into account all 20 experimental conditions (covered by 59 samples) simultaneously and allows detecting groups of genes that show similar expression patterns in an untargeted approach. The resulting network contained 3,412 genes after filtering using the coefficient of variation. The co-expressions of these genes were then fitted against a power-law model using the WGCNA package in $R$ [29]. We extracted eight modules (designated module A to module $\mathrm{H}$ ) from our network, each represented by a group of genes that share similar expression patterns (Figure 2; Additional file 8). Module sizes ranged from 109 (module G) to 1,148 genes (module B), while 139 genes could not be assigned to any module. (Additional file 9).

By using the module eigengenes [30,31] we found that modules $\mathrm{B}, \mathrm{G}$, and $\mathrm{H}$ were strongly linked to F. graminearum-inoculated samples (Additional file 10). Of these modules two exhibited a general association to all genotypes at either both time points (module B) or only 50 hai (module $\mathrm{G}$ ). Module $\mathrm{H}$ was strongly linked to the specific defense response of CM-82036 at 50 hai. Module A was also specific for CM-82036, but not for

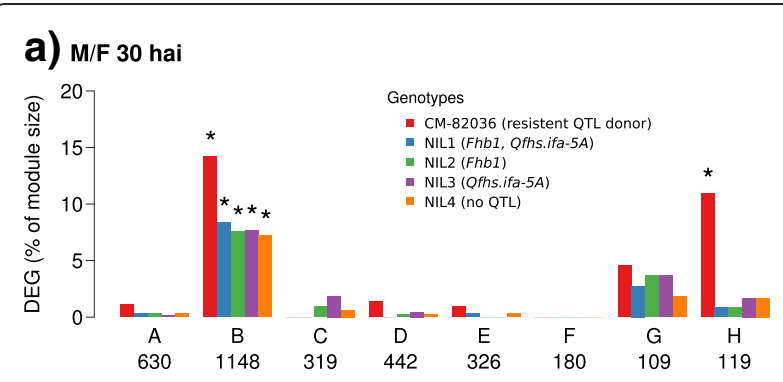

\section{b) $M / F 50$ hai}

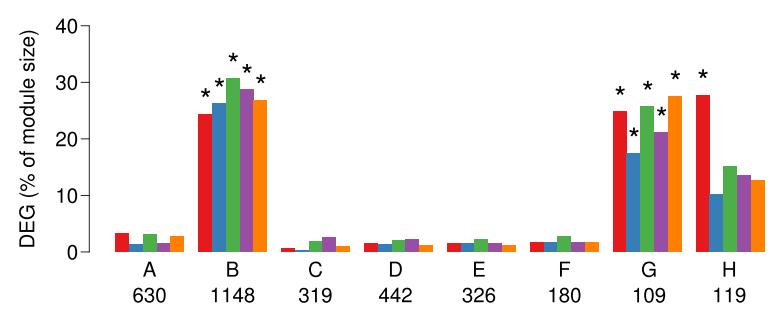

Figure 2 Differentially expressed genes per module. The bar plots indicate the ratio of $F$. graminearum responsive differentially expressed genes (DEG) per network module for 30 hours after inoculation (hai) (a) and 50 hai (b). To test whether the number of DEG genes was significantly higher than expected by chance we applied a one-sided Fisher's exact test. Stars indicate a significant enrichment at a Bonferroni adjusted p-value smaller than 0.05 . treatment or time point. A one-sided Fisher's exact test (significance threshold for Bonferroni adjusted $p$-values set to 0.05) was applied to test whether these modules showed a higher ratio of DEG than expected by chance. At 30 hai module B was strongly enriched for DEG for all five lines (Figure 2a) with CM-82036 exhibiting the highest relative amount of DEG ( $p=7.0$ e-48). This changed at 50 hai where all four NILs show a higher level of enrichment compared to CM-82036 (maximum $p=3.6$ e-65; Figure 2b). Also, 50 hai all lines exhibited a higher ratio of DEG for module $\mathrm{G}$ (maximum $p=1.7 \mathrm{e}-02$ ). Module $\mathrm{H}$ showed enrichment for CM-82036 at 30 hai $(p=1.9$ e-02) as well as 50 hai $(p=2.7 \mathrm{e}-07)$. We analyzed these data with GO and Interpro terms to obtain functional annotations for the modules. Among others, DEG in the F. graminearum responsive module $\mathrm{B}$ encoded glutathione $\mathrm{S}$-transferases (GST), UGTs, glucanases, protein kinases and WRKY transcription factors (Additional file 11). For the CM82036 related Module $\mathrm{H}$ and also for module $\mathrm{G}$ the few available GO terms did not provide sufficient meaningful annotations to predict specific molecular functions (Additional file 11).

Since module B is the by far largest module and highly enriched for $F$. graminearum responsive genes across all five lines, we further analyzed this module by splitting it into smaller submodules (deepsplit $=4$; minimum module size $=10)$. The two largest submodules comprised 475 (B-sub1) and 397 genes (B-sub2), respectively. Submodule B-sub1 was significantly enriched for DEG in all genotypes at 50 hai but only few DEG (between $5 \%$ and $10 \%$ of module size) were identified at 30 hai (Additional file 12). The relatively highest amount of DEG was found for the susceptible NIL4 and the moderately resistant NIL2. Only few GO terms were identified for this submodule (Additional file 13). B-sub2 showed a strong enrichment for DEG at 30 hai for all genotypes (minimal $p=2.8 \mathrm{e}-07$ ). This enrichment was slightly more pronounced for CM-82036, NIL1 and NIL3. These three genotypes share the resistant allele of Qfhs.ifa-5A. Consequently, B-sub2 may be associated to the activity of Qfhs.ifa-5A. The majority of GO terms for DEG in this submodule were similar to the terms identified for the pool of DEG shared by genotypes harboring Qfhs. ifa- $5 A$ (see previous section). These corresponded to kinase activity, glutamate-gated ion channels and tRNA aminoacylation (Additional file 13).

Defense-related central genes in the co-expression network A gene network allows quantifying the relative importance of single genes (nodes) by making use of local centrality measures [32-34]. Multiple methods exist for assessing the centrality of nodes. Here we applied two methods for ranking the genes by their relative importance within the network: The degree centrality ranks nodes by the number of adjacent nodes within the network, which 
allows selecting so called hub genes. These hub genes often play important roles in the regulation of gene expression and may provide valuable insight into stress response or genome evolution [35-37]. For our analysis we applied a weighted version of this measure as implemented in the igraph package [38]. Additionally, we also made use of the eigenvector centrality [39], which is related to eigenvectors of the largest eigenvalue of the adjacency matrix. To filter for the most important nodes from these two measures we used the $90 \%$ percentile and deemed nodes with values higher than this threshold as being central within the network. We will further refer to degree centrality selected genes as DCG, and to Eigenvector centrality derived genes as ECG.

In our network 218 central genes (ECG + DCG) were significantly regulated after $F$. graminearum inoculation and thus hold prominent roles in the wheat response to the pathogen. These central genes were also more likely differentially expressed in response to the pathogen than non-central genes in the network (Fisher's Exact Test, $\mathrm{BH}$-adjusted $p<0.05)$. Most belong to module $\mathrm{B}$ and only few were identified in other modules. These genes were highly enriched for GO terms associated with signaling, ubiquitination, hypersensitive response and ATP binding. The latter two are GO terms commonly used to describe NBS-LRR resistance genes, which play crucial roles in pathogen reception and signal transduction. Additional terms corresponded to nucleotide binding, suggesting the involvement of transcription factors (including WRKY, for which we found also terms in module B). Interpro annotations further identified GSTs, CYPs, glucanases and UGTs (Additional file 14). Both DCG and ECG are highly connected to other genes. Their expression behavior may have a strong impact on the global expression pattern within the network. When looking at genotype specific changes in expression of central genes, we found a group of 34 genes that were earlier differentially expressed for CM-82036 compared to the NILs. Three central genes, for which we could not retrieve annotations, were only transiently expressed. They were significantly changed for 30 hai but not 50 hai for CM-86036, while in the NILs these genes were differentially expressed only 50 hai. Yet, we detected no central genes that were only changed for CM-82036 but not for the NILs. On the other hand, 35 genes were differentially expressed exclusively for the NILs (Additional file 15). When regarding genotypes differing in the presence of either QTL, we found five central genes earlier induced for Qfhs.ifa-5A: These encode four protein kinases and a CYP. Three of these are also present in the Qhfs.ifa-5Aassociated submodule B-sub2. One of these genes XLOC_099598 encoding a protein kinase ranked third within the DCG, making it one of the highest connected central genes in our network. Similarly, we identified a
UGT, an NBS-LRR and a putative disease resistance gene as earlier induced in lines containing Fhb1.

\section{Gene family specific differential expression profiles}

The arms race between plants and pathogens has led to the rapid evolution of genes involved in the interaction with the pathogen and consequently to an increase in copy numbers to form large gene families. This allows plants to adapt to new challenges or to overcome detrimental effects of random mutagenesis by redundant gene function. The present study has among others identified glucanases, NBS-LRR proteins, WRKY transcription factors and UGTs as relevant factors in the F. graminearum/ wheat interaction - each representing a certain stage in the host defense response (recognition, signal transduction, defense regulation and toxin inactivation). To further elucidate genotype and time point specific abundance of such transcripts we expanded our analysis by taking into account the entire gene families.

We extracted Cufflinks genes encoding glucanases, NBS-LRR proteins, WRKYs and UGT using either domain specific motifs or homology information, clustered the acquired sequences using CLUSTALX N-J bootstrapping [40] and added genotype-specific DEG information (Table 2). Using this approach we identified 568 putative wheat glucanase genes via mapping against barley genes that contained the Interpro domain IPR008985. Given the hexaploid nature of wheat, a reasonable high number compared to the 262 putative glucanases in barley [20]. Similarly, we identified 246 NBS-LRR genes via mapping against 267 barley genes (www.vmatch.de), 116 WRKY transcription factors (74 in barley) via mapping against a conserved motif (WRKYGQK) and 222 putative UGTs (159 predicted functional genes in Brachypodium distachyon Beauv., [41]) by searching for the conserved signature motifs $[42,43]$.

The DEG profiles for the NBS-LRR and glucanase genes showed dramatic differences between CM-82036 and the NILs at 30 hai: 36 of 44 differentially expressed NBS-LRR genes and 83 of 112 differentially expressed glucanases were found changed for CM-82036 and about half of those genes were only changed for this genotype and not for the NILs. In contrast only 7 to 11 of the NBS-LRR and 35 to 48 of the glucanases were differentially expressed for the NILs. 50 hai all genotypes showed an equally high number of upregulated NBS-LRR and glucanase genes (up to $35 \%$ of the total number of identified NBS-LRR and glucanase genes). The dominance in gene numbers and genotype specific genes for CM-82036 at 30 hai was not observed at 50 hai. Neither for glucanases (Additional file 16), nor for NBS-LRR (Additional file 17) we observed differential expression patterns that would suggest an Fhb1- or Qfhs.ifa-5A-dependent upregulation of genes. 
Table 2 Differential expression of pathogen-induced gene families

\begin{tabular}{|c|c|c|c|c|c|c|c|c|c|}
\hline \multicolumn{10}{|c|}{30 hai } \\
\hline & Total & CM-82036 & NIL1 & NIL2 & NIL3 & NIL4 & all lines & CM-82036 only & NILs only \\
\hline glucanases & 112 & 83 & 40 & 41 & 48 & 35 & 13 & 37 & 4 \\
\hline UGTs & 36 & 31 & 15 & 13 & 20 & 15 & 7 & 13 & 2 \\
\hline WRKYs & 21 & 14 & 10 & 3 & 5 & 6 & 1 & 8 & 1 \\
\hline NBS-LRR & 44 & 36 & 8 & 9 & 11 & 7 & 2 & 26 & 0 \\
\hline \multicolumn{10}{|c|}{50 hai } \\
\hline & Total & CM-82036 & NIL1 & NIL2 & NIL3 & NIL4 & all lines & CM-82036 only & NILs only \\
\hline glucanases & 304 & 178 & 184 & 214 & 190 & 179 & 91 & 23 & 27 \\
\hline UGTs & 79 & 27 & 17 & 24 & 21 & 19 & 10 & 7 & 0 \\
\hline WRKYs & 44 & 23 & 19 & 23 & 19 & 17 & 5 & 8 & 1 \\
\hline NBS-LRR & 144 & 83 & 76 & 86 & 86 & 76 & 34 & 13 & 8 \\
\hline
\end{tabular}

Genotype specific differential expression of genes from selected gene families at 30 hours after inoculation with $F$. graminearum (hai) and 50 hai. Column 'total' describes the total number of differentially expressed genes at a certain time point. "All lines" refers to genes that were differentially expressed for all five lines.

Members of the WRKY transcription factors play a decisive role in regulating response to abiotic and biotic stresses [42]. While CM-82036 showed a stronger response at 30 hai (14 genes), we also found a relatively high number (10) of WRKY upregulated for the more the resistant NIL1 (containing Fhb1 and Qfhs-ifa.5A) compared to the moderately to susceptible NILs (NIL2-4), as depicted for 30 hai in Figure 3a and for 50 hai in Figure 3b). Potentially, the activity of both QTL leads to the (stacked) activation of multiple WRKY genes. In contrast to the NBS-LRR and glucanase gene families we found relatively less WRKY genes differentially expressed at 50 hai: No more than $20 \%$ of the 116 identified WRKY genes were differentially expressed for any genotype.

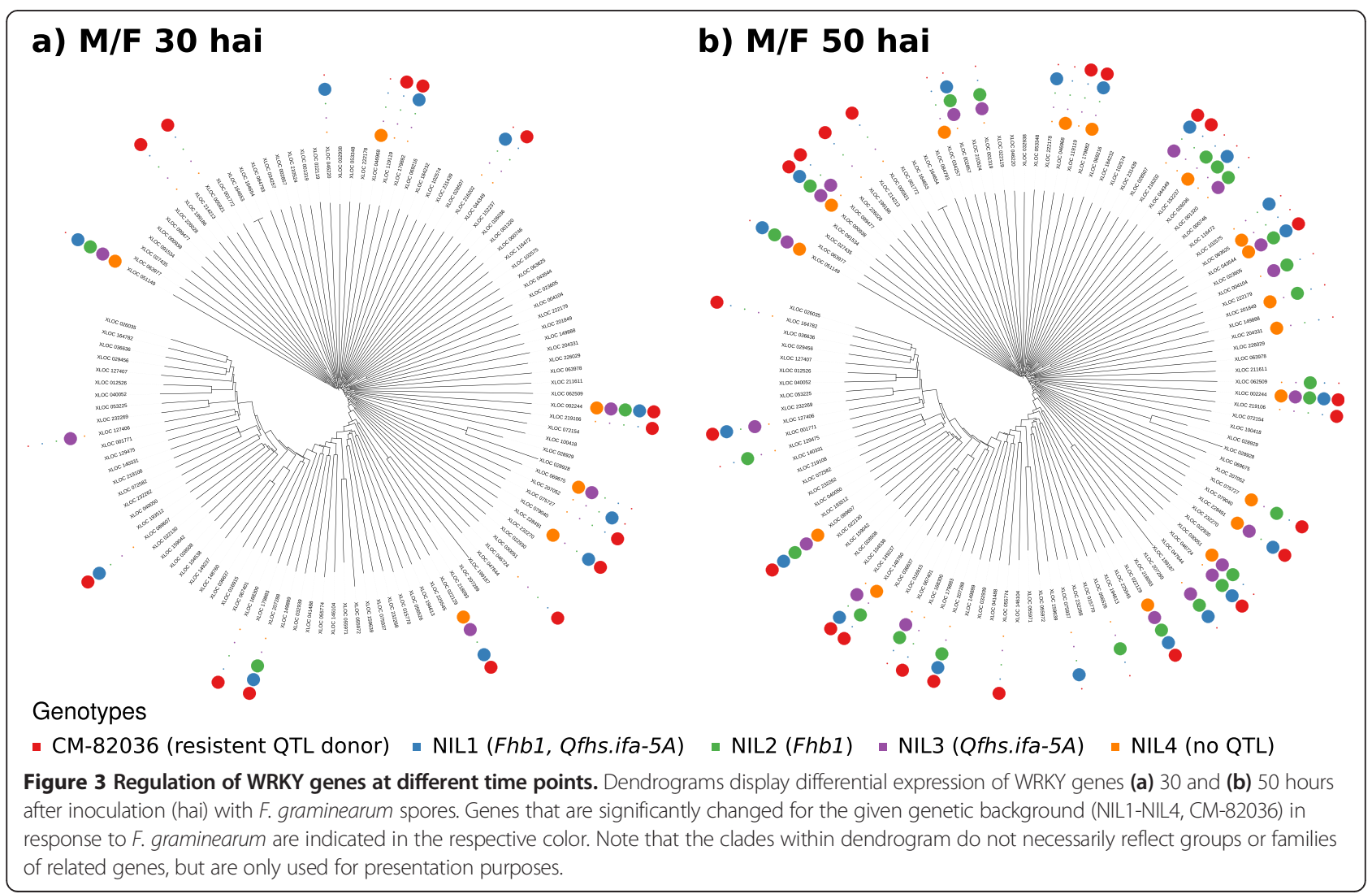


UGTs have been shown to encode the ability to inactivate the $F$. graminearum toxin DON by formation of DON-3-glucoside in Arabidopsis thaliana Heynh. (D3G, [44]) and such genes also exist in monocotyledoneous species, where they are specifically upregulated in response to the toxin $[41,45]$. Our analysis found relatively few UGTs responsive to $F$. graminearum compared to the total of 222 identified putative UGTs (Figure 4). We found no specific upregulation of UGTs in lines sharing Fhb1, the QTL associated with detoxification by glycoconjugation of the F. graminearum toxin DON ([11]). However, 50 hai, besides CM-82036 (27 differential expressed genes), NIL2, harboring Fhb1 only, exhibited the highest number of significantly changed UGTs (24). Also, while most of the DEG were found in more than just one NIL, 6 UGTs are only differentially expressed for NIL2. Recently, we have characterized a monocotyledonous UGT gene family, which encodes isozymes capable of inactivating the toxin [41]. We have identified nine putative orthologs to this gene family in our data (highlighted in Figure 4a and 4b) of which 2 were again specifically changed only for NIL2 at 50 hai (Figure $4 \mathrm{~b}$ ), while the others were either not differentially expressed at all (3) or upregulated in more than one genotype without exhibiting any QTL dependent patterns (4).

\section{Discussion}

The defense response of wheat to one of its most devastating pathogens $F$. graminearum has been investigated in multiple transcriptome profiling studies, which compared differentially resistant genotypes [46-48] or reported on the specific response to DON [49-51]. The general understanding of the wheat $/ F$. graminearum interaction has been further expanded by proteomic and metabolomic studies [12,52]. Statistics on large-scale data often rely on the detection of isolated significantly changed genes. These genes represent only a fraction of the entire defense response and potentially might not include the causative gene for mounting the resistant reaction. Here we present a co-expressed gene network, which enabled us to detect gene modules that are active in the susceptible and/or resistant genotypes. A network-driven approach has the advantage of describing coordinated gene expression changes in a holistic manner [53]. The interaction information can then be analyzed using mathematical approaches that can select features of interest for the subsequent analyses, e.g. [54,55]. Integration of DEG information into the gene co-expression network allowed for observing genotype specific dynamics in the response. In the work at hand we analyzed the effect of two major QTL by analyzing gene expression profiles of NILs segregating for either QTL.
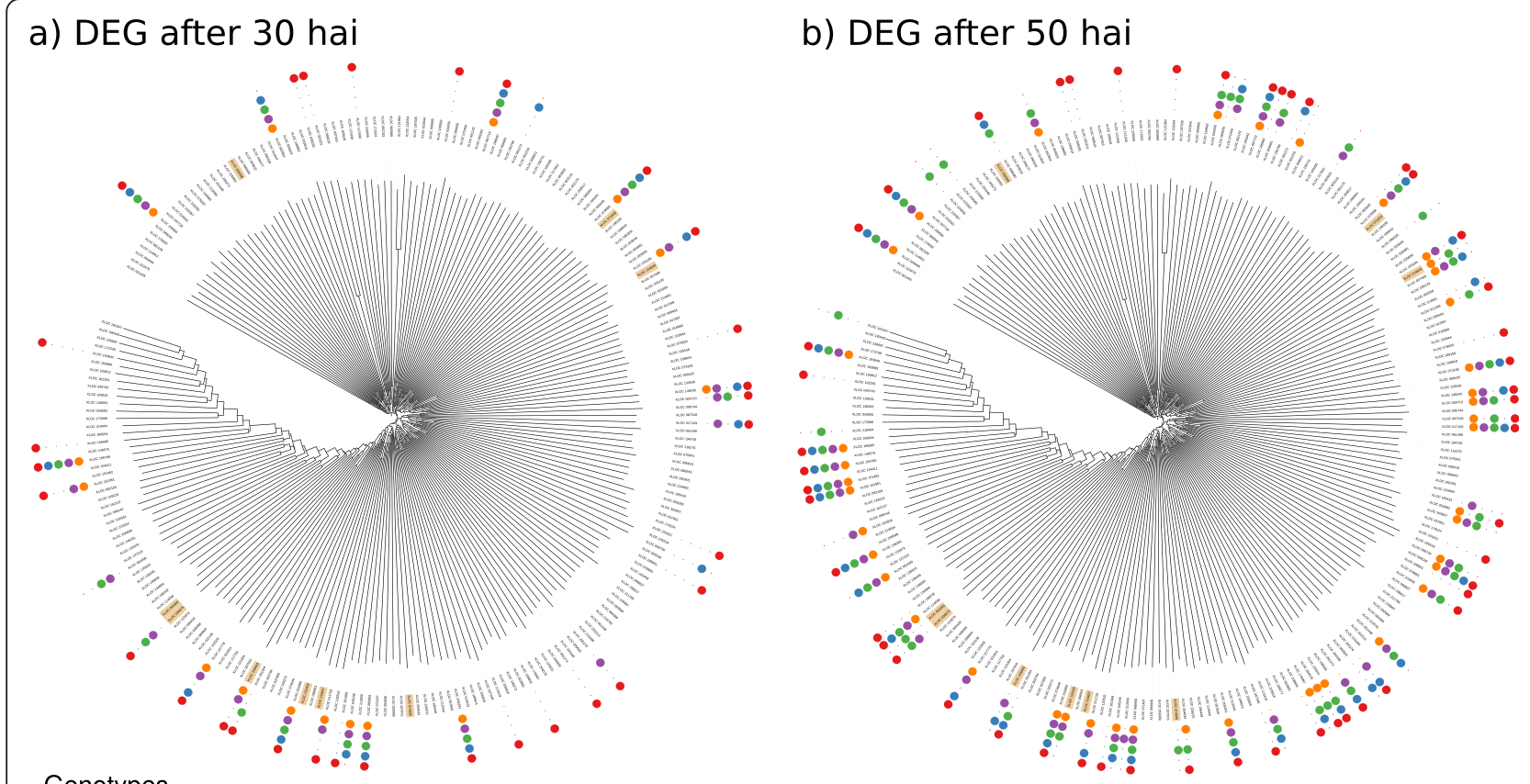

Genotypes

- CM-82036 (resistent QTL donor) - NIL1 (Fhb1, Qfhs.ifa-5A) - NIL2 (Fhb1) - NIL3 (Qfhs.ifa-5A) - NIL4 (no QTL)

Figure 4 Regulation of UGT genes at different time points. Dendrograms display differential expression of UGT genes (a) 30 and (b) 50 hours after inoculation (hai) with F. graminearum spores. Genes that are significantly changed for the given genetic background (NIL1-NIL4, CM-82036) in response to F. graminearum are indicated in the respective colour. UGT genes homologous to the previously identified DON-detoxification UGTs in B. distachyon are highlighted. Note that the clades within dendrogram do not necessarily reflect groups or families of related genes, but are only used for presentation purposes. 
The identified modules comprise groups of genes that act in concerted manner in reaction to the pathogen - and in part are also specific for genotype or the QTL. It needs however to be noted that these effects may also be influenced by the activity of closely linked, yet QTL independent genes, that have been introgressed with the QTL during the generation of the near-isogenic material. To understand the functional background of these modules it will be necessary to combine information about the genes in these modules and their interactions with additional data, which will be part of future studies. The publication of the bread wheat genome will contribute to this challenge by allowing to further split the network into subgenomespecific partitions.

The time points we chose to collect samples after inoculation with F. graminearum reflect crucial stages of the initial biotrophic growth phase of $F$. graminearum (30 hai) and in the onset of necrotrophic growth (50 hai): Germination and hyphae development occurs within 24 hai and the formation of infection hyphae has been observed at 36 hai [56]. The colonization of cells and spread into rachis and adjacent spikelet is reported to occur at about 48 hai [57]. Significant levels of DON were only found after this time point $[14,46]$. We observed two distinct defense-related modules in the gene co-expression network, which showed a time point dependent enrichment with DEG. Module B, comprising well over 1,000 genes in our network, was significantly enriched for genes upregulated in presence of the pathogen already at 30 hai and more so at 50 hai. In contrast module $\mathrm{G}$ was significantly enriched for DEG only at 50 hai. Potentially, module $G$ could reflect reaction to the transformation of the fungus into the necrotrophic stage and possibly to DON, which is not likely to be present earlier. Module $G$ was higher enriched for the more susceptible genotypes NIL2 (Fhb1) and NIL4 (no QTL) but also for CM-82036. NIL2 and NIL4 lack the resistance against initial infection conferred by $Q f h s$.ifa-5A and consequently a higher infection rate could have elicited a stronger response in these genotypes. The enrichment for CM-82036 may correspond to a general faster induction of defense mechanisms.

\section{Activation of glycolysis and amino acid biosynthesis in response to $F$. graminearum}

Our analysis finds genes involved in the pentose phosphate pathway and citric acid cycle upreguated in response to the pathogen in all genotypes. Additional terms for the pentose phosphate pathway and also for the glutathione-mediated detoxification of the toxic respiration biproduct methylglyoxal (glyoxylase I, [58]) have been found for NIL3. These findings demonstrate the elevated demand of carbohydrates and energy equivalents during the resistance response and also the probable breakdown of photosynthesis, which is required to replenish energy equivalents from carbohydrates (reviewed in [28]). The glycolysis-generated NADPH could also be used to fuel the production of reactive oxygen species. However, we do not find terms for the central enzyme in production of reactive oxygen species (ROS), NADPH oxidase in our analysis. Acquisition of the required hexoses heavily relies on the activity of cell wall invertases, which have also not been detected in our analysis. Transient silencing of a tobacco invertase, severely reduces the expression of defense-related genes [59]. However, invertase activity might not be sufficient to meet the increased requirements. The citric acid cycle can be replenished via the GABA-shunt, which utilizes glutamate as substrate [29]. We find GO terms for glutamate synthases and glutamine metabolic processes abundant already 30 hai, which could be upregulated to support the GABA-shunt. An alternative explanation suggests an indirect role in the production of secondary metabolites: Glutamine synthase have been shown upregulated in concert with phenylalanine ammonia lyases (PAL), which catalyse the transformation of phenylalanine to trans-cinnamate and represent the first dedicated step in the biosynthesis of phenylpropanoids and lignin. Ammonium is a side product of this process and may be reutilized by glutamine synthases also in order to prevent the accumulation to toxic levels.

\section{The resistant CM-82036 exerts its successful defense by reacting earlier and with a specific subnetwork}

The unique response of CM-82036 was already reflected in the high number of DEG at 30 hai in comparison to the four NILs. Also for CM-82036 a much higher number of glucanases, NBS-LRR and WRKY genes were activated earlier. The faster response in comparison to susceptible genotypes has been previously observed [57]. Not only transcript levels of putative resistance genes but also faster induction of such genes seem to be a decisive factor in mounting a successful defense response [48,52]. What distinguished the response of CM-82036 was also a unique response module (Figure 2 Module $\mathrm{H}$ ), which was not observed for any of the other lines. Besides genes involved in signaling and control of gene expression GO annotations of this module highlight terms for terpenoid and trehalose biosynthesis at 30 hai. The role of the disaccharide trehalose in plant defense has been recently reviewed [60]. Trehalose has been reported as a ROS quencher in wheat [61] and induces the expression of a WRKY6 gene and a glucanase gene in A. thaliana [28]. Treatment with trehalose confers partial protection against Blumeria graminis Speer to wheat [62]. The present study also found a considerable higher number of glucanase genes significantly changed for the earlier time point compared to the other investigated genotypes, which could relate to trehalose activity. However, it remains unclear as how the sugar exerts these functions. 


\section{A diacylglycerol kinase and early induction of JA and ET biosynthesis are associated with lines harboring Fhb1}

We have observed effects of Fhb1 in different genetic backgrounds by identifying $F$. graminearum responsive transcripts that are changed only for lines harboring the resistant Fhb1 allele. Among the few shared transcripts we found terms for $\mathrm{G}$ protein coupled signaling and diacylglycerol kinase activity (describing the same gene, 30 and 50 hai). Loss of $\mathrm{G}$ protein dependent phosphatidic acid signaling leads to reduced accumulation of defenseassociated transcripts: Plant $G$ proteins act in reception and translation of extracellular cues into intracellular second messengers. In rice the expression of the $G$ protein $\alpha$ subunit RGA1 is R-gene dependent and $r g a 1 \mathrm{mu}$ tants show a delayed production of ROS in response to Magnaporthe grisea Barr elicitors [63]. The same authors could later show that a mitogen-activated protein kinase is the downstream target of RGA1. Silencing this kinase leads to reduced levels of PR proteins and PAL [64]. A role for phosphatidic acid signaling in $F$. graminearum resistance was previously suggested by Ding and associates [52]. In this proteomic/transcriptomic study the authors find among other transcripts also diacylglycerol kinase and phospholipase D less abundant in a F. graminearumsusceptible mutant of the Fhb1-harboring resistant line Wangshuibai. Also in A. thaliana mutants of the G protein $\beta$ subunit ABG1 were shown more susceptible to a variety of fungal pathogens, including Fusarium oxysporum Schltdl. [65].

The expression of Fhb1-related genes could be coupled to disease development/presence of DON and hence to the overall resistance conferred by the respective genotype, which may delay disease development to a certain extent. The lines containing Fbh1 exhibit broad differences in FHB resistance: The highly resistant CM-82036 develops disease symptoms exclusively on the infected spikelets, while NIL1 (both QTL) and NIL2 (only Fhb1) exhibit just an intermediate level of resistance [14]. Consequently, Fhb1-associated transcripts are not necessarily significantly changed for all these lines at a given time point. To reduce complexity, we have also investigated DEG for NIL2 only, which harbors $F h b 1$ in a susceptible background. DEG encoded for proteins involved in the biosynthesis of biotic stress response hormones JA and ET already at 30 hai. Both have been implicated with resistance mediated by the QTL donor line Sumai-3 [66]. Recent work using Virus-induced gene silencing in wheat showed that plants impaired in the production of ET are more susceptible to the disease [67]. JA has also received some attention in respect of $F h b 1$ recently: The QTL was associated with a higher abundance of JA in a proteomic study using a NIL pair differing in Fhb1 [50]. In the first transcriptome sequencing study investigating an Fhb1-deletion line of Wangshuibai, the authors find no difference in the abundance of transcripts corresponding to JA biosynthesis genes, but hypothesize that JA signaling in the deletion line is impaired, since downstream targets of JA are induced in the wildtype but not in the deletion line [61]. These findings may be related to the Fhb1-associated G protein coupled kinase, which could be involved in transmitting JA signals.

Fhb1 confers resistance against spreading of the disease [9]. The QTL also co-localizes with resistance against DON - a virulence factor for F. graminearum [11] and it is very likely that both QTL relate to the same causal gene. Consequently, the QTL may exert its function only in the necrotrophic growth phase of the fungus. The resistant response in NIL2 appears delayed when observing dynamics of gene expression for four closer characterized gene families (Figures 3 and 4, Additional files 16 and 17). While CM-82036 exhibits the highest number of DEG at 30 hai for glucanase, NBS-LRR, WRKY and UGT genes, NIL2 and NIL4 show fewer DEG than the other lines at this time point. At 50 hai many more genes are upregulated for all genotypes (Table 2). By then, NIL2 has caught up and exhibits a similar strong response as CM-82036, while NIL4 still contains among the lowest number of DEG. The stronger response for NIL2 at 50 hai, could suggest the requirement of additional environmental cues such as penetration of host tissue/DON accumulation, which may not be present at 30 hai. Especially the UGT gene family is interesting in respect of Fhb1: Lines harboring the QTL contain a higher ratio of the UGT-mediated DON detoxification product DON-3-glucoside [11]. Wheat UGTs orthologous to a recently described gene cluster of B. distachyon [41] harboring DON-detoxification UGTs potentially share this ability. Our analysis identified 9 close homologs, which are likely candidates for future functional analysis. 2 and 7 of these homologs are expressed 30 and 50 hai, respectively, which is in line with the observation that these UGTs are not induced by $F$. graminearum but specifically for the toxin [41]. However, only few genes are specifically induced in lines with Fhb1 or NIL2 only.

\section{Lines harboring Qfhs.ifa-5A exhibited higher activity in defense module B-sub2 and were associated with calcium signaling and riboflavin biosynthesis}

In contrast to Fhb1, Qfhs.ifa-5A confers type I resistance against initial infection of $F$. graminearum [9]. Although the infection method used in this study favors the phenotypic assessment of type II resistance, the resistance mediated by Qfhs.ifa-5A can be assessed using this technique, and consequently also the QTL-specific transcriptional response may be captured. In this study we found more genes in the defense-associated network module B-sub2 differentially expressed for lines harboring Qfhs.ifa-5A at 30 hai (CM-82036, NIL1, NIL3) than for lines without the QTL. While this may not represent 
a QTL-specific gene subnetwork, we suggest that these genes were faster or stronger differentially expressed for these lines due to the activity of the Qfhs.ifa-5A. We identified a group of central genes encoding protein kinases and a CYP gene within this module, which were earlier induced for Qfhs.ifa-5A lines. These all are likely candidates for future functional analysis. Submodule B-sub2 comprised 397 genes and included kinases activity, glutamate-gated ion channels and genes involved in tRNA processing (Additional file 13). Elevated tRNA abundance has been previously linked to the response to DON in barley [68] and other abiotic stresses [69]. Higher translational activity could be a secondary effect to the toxin and not an active resistance response. Since, tRNA related terms are only found in the core set of DEG shared by all genotypes (Figure 1), we conclude that these most likely are not related to the resistance conferred by Qfhs.ifa-5A. In contrast, the glutamate-gated ion channel identified in the submodule was also changed only for lines sharing the QTL in the DEG analysis at 30 hai. Endogenous or environmental factors trigger changes in apoplasmatic glutamate concentration, which leads to the activation of these channels and subsequently to an intracellular increase of $\mathrm{Ca}^{2+}[70] . \mathrm{Ca}^{2+}$ influx is associated with early defense signalling [71]. Overexpression of ionotrophic glutamate receptors in $A$. thaliana leads to an increase in $\mathrm{Ca}^{2+}$ influx and consequently to a delayed infection with Botrytis cinerea Pers. [72]. Downstream targets of $\mathrm{Ca}^{2+}$ signaling such as ATPases or calmodulin are frequently reported as induced by F. graminearum (e.g. [52]). Genotypes harboring Qfhs.ifa-5A also share GO terms relating to the biosynthesis and the metabolism of riboflavin (30 hai). Riboflavin has been reported to induce resistance to fungal and other pathogens [73], potentially by recruiting NPR1, the essential regulator of systemic acquired resistance, independently from the defense-signaling hormone salicylic acid, which is strongly associated with NPR1 [74]. Riboflavin is also implicated in the activation of ethylene biosynthesis [73] and we also find genes related to ethylene biosynthesis in the section shared by lines harboring Qfhs.ifa-5A (Additional file 6, 30 hai).

Taken together this study provides insights into resistance response of differentially resistant wheat genotypes to F. graminearum. By combining a gene co-expression network approach with differential gene expression analysis we were able to make observation of genes and pathways associated with two prominent resistance QTL, Fhb1 and Qfhs.ifa-5A. Central genes within the network may be valid candidate genes for functional testing.

\section{Conclusions}

This RNA-seq study provides insights into the QTLdependent defense response of bread wheat against
F. graminearum. We find G-protein coupled receptor kinases and biosynthesis genes for jasmonate and ethylene earlier induced for NILs harboring Fhb1 and genes involved in the biosynthesis and metabolism of riboflavin were found more abundant after infection in lines harboring Qfhs.ifa-5A. By combining a gene coexpression network approach with differential gene expression analysis we identified genes and pathways associated with the investigated NILs and the resistant parent CM-82036. Central genes within the network may be promising candidate genes for functional testing. Revisiting these and other data after the complete wheat genes are available will provide even higher resolved insights into the defense response dynamics within the gene co-expression network.

\section{Methods}

\section{Plant material and inoculation experiment}

Four NILs previously generated from a cross of the resistant spring wheat line CM-82036 and Remus, a susceptible German spring wheat cultivar [14] were investigated in these experiments and also the resistant parent CM82036. The NILs have been developed from one BC5F1 plant with Remus as the recurrent parent (5 backcrosses). In the BC5F2 lines that contain the resistance alleles from CM-82036 of both Fhb1 and Qfhs.ifa-5A (NIL1), or either Fhb1 (NIL2) or Qfhs.ifa-5A (NIL3) or none (NIL4) have been selected. F. graminearum conidia spores required for inoculation were produced on defined SNA medium under UV-light at $25^{\circ} \mathrm{C}$. After two weeks conidia were harvested and diluted to 50,000 conidia/mL in water. Aliquots were stored at $-80^{\circ} \mathrm{C}[10]$.

Plant growth conditions and the inoculation of flowering plants with F. graminearum spores were described previously [14]. Briefly, 12 florets per head (from 6 central spikelets, the two basal florets) were inoculated at anthesis with $10 \mu \mathrm{l}$ of a F. graminearum conidia spore suspension (500 conidia, concentration 50.000 conidia / mL) or mock by cautiously inserting a droplet onto the generative part of each floret without wounding the tissue. The treated heads were moistened with water and covered in plastic bags for 24 hours to provide humid conditions favorable for infection with the pathogen. Only palea and lemma of the inoculated florets were sampled including the respective part of the rachis. For each of the 60 samples (five genotypes, F. graminearum/mock treatment, two time points 30 and 50 hai, three replicates) 12 heads were used and pooled into one combined sample and stored at $-80^{\circ} \mathrm{C}$ until use.

\section{RNA-extraction and sequencing}

To eliminate RNases, metal jars with inherent metal spheres for Retsch-mill (MM 301, Haan, Germany) were sterilized at $180^{\circ} \mathrm{C}$ for $3 \mathrm{~h}$ and then stored at $-80^{\circ} \mathrm{C}$. All 
tissue belonging to one sample was pooled in one precooled jar and clamped in Retsch-mill. Grinding was performed for 30 seconds at full speed to obtain a fine tissue powder and immediately put back at $-80^{\circ} \mathrm{C}$. TotalRNA was extracted from $100 \mathrm{mg}$ of frozen tissue powder using the RNeasy Plant Mini Kit (\#74903, Qiagen, Venlo, Netherlands) according to manufacturer's instructions. The extracted RNA was checked for quality and quantity on an automated electrophoresis-system (Experion, \#7017000, Bio-Rad, Hercules, CA, US). Sequencing was performed on an Illumina HiSeq2000 machine using $8 x$ multiplexing, theoretically generating $22 \mathrm{M}$ reads per sample by the sequencing-provider GATC (Konstanz, Germany). The respective data sets are available in the EBI ArrayExpress (www.ebi.ac.uk/arrayexpress/) repository under the accession number E-MTAB-1729.

\section{Data processing and mapping}

The recently published LCG wheat assembly [5] was used to identify RNA-Seq transcripts with Tophat and Cufflinks [24]. We removed one mock-treatment sample from genotype NIL2 (50 hai) as it did not pass quality control. Transcripts were combined with Cuffcompare and were mapped against barley high confidence genes [18] with Vmatch (www.vmatch.de) (exdro $p=3$, seedlength $=12$, hit length $=100 \mathrm{bp}$, identity $>85 \%$ ) requiring a $\mathrm{BBH}$ and by taking the longest transcript of each reported gene loci. Transcripts with existing $\mathrm{BBH}$ to a barley gene served as input for network analysis and enrichment analyses.

\section{Statistical analysis of differential gene expression}

Differentially expressed genes (DEG) were detected with Cuffdiff [24] between treatments in pair-wise comparisons. These were performed for samples from the same genotype but for different treatments or time-points. Thus we compared mock-inoculation against Fusarium-inoculation $(\mathrm{M} / \mathrm{F})$ at 30 hai and 50 hai. Additionally, we compared mock-inoculation at 30 hai with 50 hai (M/M). Cuffdiff default parameters were applied and the FDR-adjusted $p$-value was taken as a cut-off, keeping values below 0.1 as DEG. Genes that were significantly differentially expressed in $\mathrm{M} / \mathrm{M}$ were not considered as differentially expressed in $\mathrm{M} / \mathrm{F}$ comparison and vice versa.

\section{Co-expression network and module detection}

Gene expression values (FPKM) were taken to infer a gene co-expression network. We used the $\log _{2}$-transformed FPKM values, and replaced values smaller than one by zero. In order to keep only the most active genes we applied a coefficient of variation filter with a threshold of 1 across the different conditions. In order to infer a network, we made use of the WGCNA package [29] with the softthresholding parameter beta set to 4 and absolute Pearson's correlation coefficient. Thereby, we inferred an undirected, weighted network. Within the network, clusters of genes with similar expression patterns, so called modules, were then inferred using a clustering of the Topological Overlay Matrix (cutreeDynamic method; deepSplit $=2$, minimal module size $=40$, merging similar modules with parameter cut height $=0.2$ ). The module eigengene (ME, [30]) of a given module is defined by the first principal component of the module expression matrix and can be regarded as the representative of the gene expression in a module [31]. By using the ME we could quantify the association between a module and the samples, with larger values indicating a stronger association with a module.

\section{GO and interpro enrichment analyses}

$\mathrm{GO}$ terms and Interpro domains for the BBH transcripts were extracted from the barley repository (ftp://ftpmips. gsf.de/plants/barley/public_data/). GO enrichment analyses were performed using the topGO package [26], using Fisher's exact test. To reduce the number of false positive findings the elim algorithm was applied and reported $p$-values smaller than 0.05 were kept for further analyses. Interpro terms were tested for enrichment using one-sided Fisher's exact test, keeping Benjamini-Hochberg [25] adjusted $p$-values smaller than 0.05 .

\section{Additional files}

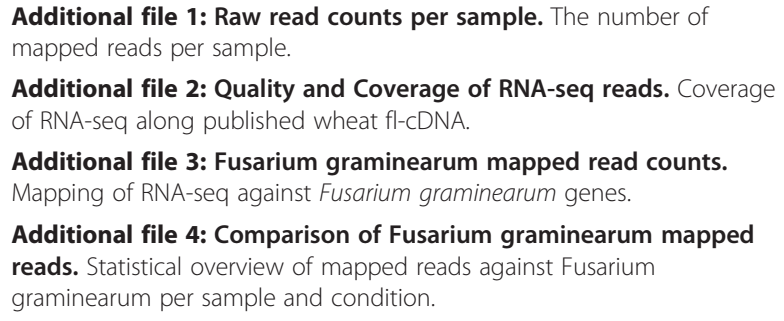

Additional file 1: Raw read counts per sample. The number of mapped reads per sample.

Additional file 2: Quality and Coverage of RNA-seq reads. Coverage of RNA-seq along published wheat fl-cDNA.

Additional file 3: Fusarium graminearum mapped read counts. Mapping of RNA-seq against Fusarium graminearum genes.

Additional file 4: Comparison of Fusarium graminearum mapped reads. Statistical overview of mapped reads against Fusarium graminearum per sample and condition.

Additional file 5: List of differentially expressed genes. A summary of differentialy expressed genes.

Additional file 6: Enrichment analyses of DEG at $\mathbf{3 0}$ hai. Results of GO and Interpro enrichment analyses for DEG at 30 hai.

Additional file 7: Enrichment analyses of DEG at $\mathbf{5 0}$ hai. Results of GO and Interpro enrichment analyses for DEG at 50 hai.

Additional file 8: Module inference. Colored dendrogram showing the modules as inferred from the co-expression network.

Additional file 9: Gene module membership. The module membership of the analyzed genes as well as the contig mapping information.

Additional file 10: Module eigengenes. Summary of the module eigengenes.

Additional file 11: Enrichment analyses for modules. Results of $G O$ and Interpro enrichment analyses for modules $B, G$, and $H$.

Additional file 12: DEG in submodules of module B. Similar to Figure 2 the ratio of DEG in the submodules of module $B$ is depicted. Additional file 13: Enrichment analyses for modules of module B. Results of GO and Interpro enrichment analyses for module B submodules 1 and 2 .

Additional file 14: Enrichment analyses for central genes. Results of GO and Interpro enrichment analyses for central genes. 
Additional file 15: Differentially expressed central genes.

Differentially expressed central genes and annotations with frequently observed $\mathrm{GO}$ terms.

Additional file 16: Regulation of NBS-LRR genes at different time points. The line-specific regulation of NBS-LRR genes at different time points.

Additional file 17: Regulation of glucanases at different time points. The line-specific regulation of glucanases at different time points.

\section{Competing interests}

The authors declare no competing interests.

\section{Authors' contributions}

$\mathrm{KK}, \mathrm{KM}$, WS, HB designed the research; KK, GS, TN, BS, ML performed the research; KK, TN, CA, MS, WS analyzed the data; KK, GS, TN, WS wrote the paper. All authors read and approved the final manuscript.

\section{Acknowledgements}

We thank Mina Samad Zamini, Morgane Roth, Erik Sam, and Maria Buerstmayr for assisting in the climatic chamber experiments and Imer Maloku for the preparation of the F. graminearum inoculum. This work was supported by the Austrian Science Fund projects \#F3711-B11 and \#F3711-B05.

\section{Supporting data}

The data set supporting the results of this article is available in the ArrayExpress repository (www.ebi.ac.uk/arrayexpress), accession number E-MTAB-1729.

\section{Author details}

'Munich Information Center for Protein Sequences/Institute for Bioinformatics and Systems Biology, Helmholtz Center Munich, D-85764 Neuherberg, Germany. ${ }^{2}$ Institute for Biotechnology in Plant Production, IFATulln, University of Natural Resources and Life Sciences, A-3430 Tulln, Austria.

Received: 25 June 2013 Accepted: 14 October 2013

Published: 24 October 2013

\section{References}

1. Cuomo CA, Guldener U, Xu JR, Trail F, Turgeon BG, Di Pietro A, Walton JD, Ma LJ, Baker SE, Rep M, Adam G, Antoniw J, Baldwin T, Calvo S, Chang Y-L, DeCaprio D, Gale KR, Gnerre S, Goswami RS, Hammond-Kosack K, Harris LJ, Hilburn K, Kennell JC, Kroken S, Magnuson JK, Mannhaupt G, Mauceli E, Mewes H-W, Mitterbauer R, Muehlbauer G, et al: The Fusarium graminearum genome reveals a link between localized polymorphism and pathogen specialization. Science 2007, 317:1400-1402.

2. Walter S, Nicholson P, Doohan FM: Action and reaction of host and pathogen during Fusarium head blight disease. New Phytol 2010 185:54-66.

3. Pestka JJ: Deoxynivalenol: mechanisms of action, human exposure, and toxicological relevance. Arch Toxicol 2010, 84:663-679.

4. The European Commission: Commission recommendation (EC) no. 1881/ 2006 of 19 December 2006 setting maximum levels for certain contaminants in foodstuffs. Off J Eur Union 2006, 364:5-24

5. Brenchley R, Spannagl M, Pfeifer M, Barker GL, D'Amore R, Allen AM, McKenzie N, Kramer M, Kerhornou A, Bolser D, Kay S, Waite D, Trick M, Bancroft I, Gu Y, Huo N, Luo M-C, Sehgal S, Gill B, Kianian S, Anderson O, Kersey P, Dvorak J, McCombie WR, Hall A, Mayer FKX, Edwards KJ, Bevan MW, Hall N: Analysis of the bread wheat genome using whole-genome shotgun sequencing. Nature 2012, 491:705-710.

6. Paux E, Roger D, Badaeva E, Gay G, Bernard M, Sourdille P, Feuillet C Characterizing the composition and evolution of homoeologous genomes in hexaploid wheat through BAC-end sequencing on chromosome 3B. Plant J 2006, 48:463-474.

7. Buerstmayr H, Ban T, Anderson JA: QTL mapping and marker-assisted selection for fusarium head blight resistance in wheat: a review. Plant Breed 2009, 128:1-26.

8. Waldron BL, Moreno-Sevilla B, Anderson JA, Stack RW, Frohberg RC: RFLP mapping of QTL for fusarium head blight resistance in wheat. Crop SCi 1999, 39:805-811.

9. Buerstmayr H, Lemmens M, Hartl L, Doldi L, Steiner B, Stierschneider M, Ruckenbauer P: Molecular mapping of QTLs for fusarium head blight resistance in spring wheat. I. Resistance to fungal spread (Type II resistance). Theor App/ Genet 2002, 104:84-91.

10. Buerstmayr H, Steiner B, Hartl L, Griesser M, Angerer N, Lengauer D, Miedaner T, Schneider B, Lemmens M: Molecular mapping of QTLs for fusarium head blight resistance in spring wheat. II. Resistance to fungal penetration and spread. Theor Appl Genet 2003, 107:503-508.

11. Lemmens M, Scholz U, Berthiller F, Dall'Asta C, Koutnik A, Schuhmacher R, Adam G, Buerstmayr H, Mesterhazy A, Krska R, Ruckenbauer P: The ability to detoxify the mycotoxin deoxynivalenol colocalizes with a major quantitative trait locus for fusarium head blight resistance in wheat. Mol Plant-Microbe Interact 2005, 18:1318-1324.

12. Gunnaiah R, Kushalappa AC, Duggavathi R, Fox S, Somers DJ: Integrated metabolo-proteomic approach to decipher the mechanisms by which wheat QTL (Fhb1) contributes to resistance against Fusarium graminearum. PloS one 2012, 7:e40695.

13. Zhuang Y, Gala A, Yen Y: Identification of functional genic components of major Fusarium head blight resistance quantitative trait loci in wheat cultivar Sumai 3. Mol Plant-Microbe Interact 2013, 26:442-450.

14. Schweiger W, Steiner B, Ametz C, Siegwart G, Wiesenberger G, Berthiller F, Lemmens M, Jia H, Adam G, Muehlbauer GJ, Kreil DP, Buerstmayr H: Transcriptomic characterization of two major fusarium resistance QTL, Fhb1 and Qfhs.ifa-5A, identifies novel candidate genes. Mol Plant Pathol 2013, 14:772-785.

15. Schreiber A, Hayden M, Forrest K, Kong S, Langridge P, Baumann U: Transcriptome-scale homoeolog-specific transcript assemblies of bread wheat. BMC Genom 2012, 13:492.

16. Mochida K, Yoshida T, Sakurai T, Ogihara Y, Shinozaki K: TriFLDB: a database of clustered full-length coding sequences from Triticeae with applications to comparative grass genomics. Plant Physiol 2009, 150:1135-1146.

17. Kubalakova M, Vrana J, Cihalikova J, Simkova H, Dolezel J: Flow karyotyping and chromosome sorting in bread wheat (Triticum aestivum L.). Theor Appl Genet 2002, 104:1362-1372.

18. Poland JA, Brown PJ, Sorrells ME, Jannink JL: Development of high-density genetic maps for barley and wheat using a novel two-enzyme genotyping-by-sequencing approach. PloS one 2012, 7:e32253.

19. Philippe $R$, Choulet $F$, Paux E, van Oeveren J, Tang J, Wittenberg AH, Janssen A, van Eijk MJ, Stormo K, Alberti A, Wincker P, Akhunov E, van der Vossen $E$, Feuillet $C$ : Whole genome profiling provides a robust framework for physical mapping and sequencing in the highly complex and repetitive wheat genome. BMC genom 2012, 13:47.

20. The International Barley Genome Sequencing Consortium: A physical, genetic and functional sequence assembly of the barley genome. Nature 2012, 491:711-716.

21. Adhikari BN, Savory EA, Vaillancourt B, Childs KL, Hamilton JP, Day B, Buell CR: Expression profiling of Cucumis sativus in response to infection by Pseudoperonospora cubensis. PloS one 2012, 7:e34954.

22. Bagnaresi $P$, Biselli $C$, Orru L, Urso S, Crispino L, Abbruscato P, Piffanelli $P$, Lupotto $E$, Cattivelli $L$, Vale G: Comparative transcriptome profiling of the early response to Magnaporthe oryzae in durable resistant vs susceptible rice (Oryza sativa L.) genotypes. PloS one 2012, 7:e51609.

23. Wong P, Walter M, Lee W, Mannhaupt G, Munsterkotter M, Mewes HW, Adam G, Guldener U: FGDB: revisiting the genome annotation of the plant pathogen fusarium graminearum. Nuc Acids Res 2011, 39:D637-D639.

24. Trapnell C, Roberts A, Goff L, Pertea G, Kim D, Kelley DR, Pimentel H, Salzberg SL, Rinn JL, Pachter L: Differential gene and transcript expression analysis of RNA-seq experiments with TopHat and cufflinks. Nat Protoc 2012, 7:562-578

25. Benjamini $Y$, Hochberg $Y$ : Controlling the false discovery rate: a practical and powerful approach to multiple testing. J R Stat Soc Ser B Stat Methodol 1995, 57:289-300.

26. Alexa A, Rahnenführer J, Lengauer T: Improved scoring of functional groups from gene expression data by decorrelating $\mathrm{GO}$ graph structure. Bioinformatics 2006, 22:1600-1607.

27. Bolton MD: Primary metabolism and plant defense-fuel for the fire. Mol Plant-Microbe Interact 2009, 22:487-497.

28. Seifi HS, Van Bockhaven J, Angenon G, Hofte M: Glutamate metabolism in plant disease and defense: friend or foe? Mol Plant-Microbe Interact 2013 26:475-485. 
29. Langfelder $\mathrm{P}$, Horvath S: WGCNA: an R package for weighted correlation network analysis. BMC bioinformatics 2008, 9:559.

30. Langfelder $P$, Horvath $\mathrm{S}$ : Eigengene networks for studying the relationships between co-expression modules. BMC Syst Biol 2007, 1:54.

31. Langfelder $P$, Luo R, Oldham MC, Horvath $S$ : Is my network module preserved and reproducible? PLoS Comput Biol 2011, 7:e1001057.

32. Freeman L: Centrality in social networks: conceptual clarification. Soc Networks 1979, 1:215-239.

33. Wasserman SFK: Social network analysis : methods and applications. Cambridge, New York: Cambridge University Press; 1994.

34. Hage P, Harary F: Eccentricity and centrality in networks. Soc Networks 1995, 17:57-63.

35. Jeong H, Mason SP, Barabasi AL, Oltvai ZN: Lethality and centrality in protein networks. Nature 2001, 411:41-42

36. Filteau M, Pavey SA, St-Cyr J, Bernatchez L: Gene coexpression networks reveal key drivers of phenotypic divergence in lake whitefish. Mol Biol Evol 2013, 30:1384-1396.

37. Ferreira RM, Rybarczyk-Filho JL, Dalmolin RJ, Castro MA, Moreira JC, Brunnet $L G$, de Almeida RM: Preferential duplication of intermodular hub genes: an evolutionary signature in eukaryotes genome networks. Plos one 2013, 8:e56579.

38. Csardi G, Nepusz T: The igraph software package for complex network research. InterJournal 2006, Complex Systems:1695.

39. Bonacich P: Power and centrality: a family of measures. Am J Sociol 1987 92:1170-1182.

40. Larkin MA, Blackshields G, Brown NP, Chenna R, McGettigan PA, McWilliam $H$, Valentin F, Wallace IM, Wilm A, Lopez R, Thompson JD, Gibson TJ, Higgins DG: Clustal w and clustal x version 2.0. Bioinformatics 2007, 23:2947-2948.

41. Schweiger W, Pasquet JC, Nussbaumer T, Kovalsky Paris MP, Wiesenberger G, Macadre C, Ametz C, Berthiller F, Lemmens M, Saindrenan P, Mewes HW, Mayer KF, Dufresne M, Adam G: Functional characterization of two clusters of Brachypodium distachyon UDP-glycosyltransferases encoding putative deoxynivalenol detoxification genes. Mol Plant-Microbe Interact 2013, 26:781-792.

42. Eulgem T, Rushton PJ, Robatzek S, Somssich IE: The WRKY superfamily of plant transcription factors. Trends Plant Sci 2000, 5:199-206.

43. Vogt $T$, Jones P: Glycosyltransferases in plant natural product synthesis: characterization of a supergene family. Trends Plant Sci 2000, 5:1360-1385.

44. Poppenberger B, Berthiller F, Lucyshyn D, Sieberer T, Schuhmacher R, Krska R, Kuchler K, Glossl J, Luschnig C, Adam G: Detoxification of the fusarium mycotoxin deoxynivalenol by a UDP-glucosyltransferase from Arabidopsis thaliana. J Biol Chem 2003, 278:47905-47914.

45. Schweiger W, Boddu J, Shin S, Poppenberger B, Berthiller F, Lemmens M, Muehlbauer GJ, Adam G: Validation of a candidate deoxynivalenolinactivating UDP-glucosyltransferase from barley by heterologous expression in yeast. Mol Plant-Microbe Interact 2010, 23:977-986.

46. Jia H, Cho S, Muehlbauer GJ: Transcriptome analysis of a wheat near-isogenic line pair carrying fusarium head blight-resistant and -susceptible alleles. Mol Plant-Microbe Interact 2009, 22:1366-1378.

47. Foroud NA, Ouellet $T$, Laroche A, Oosterveen B, Jordan MC, Ellis BE, Eudes F: Differential transcriptome analyses of three wheat genotypes reveal different host response pathways associated with fusarium head blight and trichothecene resistance. Plant Pathol 2012, 61:296-314.

48. Gottwald S, Samans B, Luck S, Friedt W: Jasmonate and ethylene dependent defence gene expression and suppression of fungal virulence factors: two essential mechanisms of fusarium head blight resistance in wheat? BMC genomics 2012, 13:369.

49. Walter S, Brennan J, Trognitz F, Trognitz B, Leonard G, Egan D, Doohan F Components of the gene network associated with genotype-dependent response of wheat to the fusarium mycotoxin deoxynivalenol. Funct Integr Genomics 2008, 8:421-427.

50. Lulin M, Yi S, Aizhong C, Zengjun Q, Liping X, Peidu C, Dajun L, Xiu EW: Molecular cloning and characterization of an up-regulated UDPglucosyltransferase gene induced by DON from Triticum aestivum L. cv. Wangshuibai. Mol Biol Rep 2010, 37:785-795.

51. Ansari KI, Walter S, Brennan JM, Lemmens M, Kessans S, McGahern A, Egan D, Doohan FM: Retrotransposon and gene activation in wheat in response to mycotoxigenic and non-mycotoxigenic-associated fusarium stress. Theor Appl Genet 2007, 114:927-937.
52. Ding $L, X u H, Y i H$, Yang L, Kong Z, Zhand L, Xue S, Jia H: Resistance to hemi-biotrophic $F$. graminearum infection is associated with coordinated and ordered expression of diverse defense signaling pathways. PloS one 2011, 6:e19008.

53. Emmert-Streib F, Dehmer M: Networks for systems biology: conceptual connection of data and function. IET Syst Biol 2011, 5:185-207.

54. Todeschini R, Consonni V: Handbook of Molecular Descriptors. Weilheim: WILEY-VCH; 2008

55. Mueller LA, Kugler KG, Dander A, Graber A, Dehmer M: QuACN: an R package for analyzing complex biological networks quantitatively. Bioinformatics 2011, 27:140-141.

56. Seong KY, Zhao X, Xu JR, Guldener U, Kistler HC: Conidial germination in the filamentous fungus Fusarium graminearum. Fung Genet Biol 2008, 45:389-399.

57. Pritsch C, Muehlbauer GJ, Bushnell WR, Somers DA, Vance CP: Fungal development and induction of defense response genes during early infection of wheat spikes by Fusarium graminearum. Mol Plant-Microbe Interact 2000, 13:159-169.

58. Dixon DP, Cummins I, Cole DJ, Edwards R: Glutathione-mediated detoxification systems in plants. Curr Opin Plant Biol 1998, 1:258-266.

59. Essmann J, Schmitz-Thom I, Schon H, Sonnewald S, Weis E, Scharte J: RNA interference-mediated repression of cell wall invertase impairs defense in source leaves of tobacco. Plant physiol 2008, 147:1288-1299.

60. Fernandez O, Bethencourt L, Quero A, Sangwan RS, Clement C: Trehalose and plant stress responses: friend or foe? Trends Plant Sci 2010 15:409-417.

61. Luo Y, Li W-M, Wang W: Trehalose: protector of antioxidant enzymes or reactive oxygen species scavenger under heat stress? Environ Exper Bot 2008, 63:378-384.

62. Renard-Merlier $D$, Randoux B, Nowak E, Farcy F, Durand R, Reignault $P$ : lodus 40 , salicylic acid, heptanoyl salicylic acid and trehalose exhibit different efficacies and defence targets during a wheat/powdery mildew interaction. Phytochem 2007, 68:1156-1164.

63. Suharsono U, Fujisawa Y, Kawasaki T, Iwasaki Y, Satoh H, Shimamoto K: The heterotrimeric $\mathrm{G}$ protein alpha subunit acts upstream of the small GTPase Rac in disease resistance of rice. Proc Natl Acad Sci U S A 2002, 99:13307-13312.

64. Lieberherr D, Thao NP, Nakashima A, Umemura K, Kawasaki T, Shimamoto K: A sphingolipid elicitor-inducible mitogen-activated protein kinase is regulated by the small GTPase OsRac1 and heterotrimeric G-protein in rice 1[w]. Plant Physiol 2005, 138:1644-1652.

65. Trusov Y, Rookes JE, Chakravorty D, Armour D, Schenk PM, Botella JR: Heterotrimeric $G$ proteins facilitate arabidopsis resistance to necrotrophic pathogens and are involved in jasmonate signaling. Plant Physiol 2006, 140:210-220.

66. Li G, Yen $Y$ : Jasmonate and ethylene signaling pathway may mediate fusarium head blight resistance in wheat. Crop Sci 2008, 48:1888-1896.

67. Gillespie ME, Brandt AS, Scofield SR: Ethylene-signaling is essential for basal resistance to Fusarium head blight in wheat. In Proceedings of the National Fusarium Head Blight Forum: 4-6 December 2012; Orlando, FL. Edited by Canty S. East Lansing, MI/Lexington, KY: US Wheat \& Barley Scab Initiative; 2012:135

68. Gardiner S, Boddu J, Berthiller F, Hametner C, Stupar R, Adam G, Muehlbauer G: Transcriptome analysis of the barley-deoxynivalenol interaction: evidence for a role of glutathione in deoxynivalenol detoxification. Mol Plant-Microbe Interact 2010, 23:962-976.

69. Wu XR, Kenzior A, Willmot D, Scanlon S, Chen Z, Topin A, He SH, Acevedo A, Folk WR: Altered expression of plant lysyl tRNA synthetase promotes tRNA misacylation and translational recoding of lysine. Plant J 2007, 50:627-636.

70. Dennison KL, Spalding EP: Glutamate-gated calcium fluxes in arabidopsis. Plant Physiol 2000, 124:1511-1514

71. Lecourieux D, Mazars C, Pauly N, Ranjeva R, Pugin A: Analysis and effects of cytosolic free calcium increases in response to elicitors in Nicotiana plumbaginifolia cells. Plant Cell 2002, 14:2627-2641.

72. Kang S, Kim HB, Lee H, Choi JY, Heu S, Oh CJ, Kwon Sl, An CS: Overexpression in arabidopsis of a plasma membrane-targeting glutamate receptor from small radish increases glutamate-mediated $\mathrm{Ca}^{2+}$ influx and delays fungal infection. Mol Cells 2006, 21:418-427. 
73. Dong H, Beer SV: Riboflavin induces disease resistance in plants by activating a novel signal transduction pathway. Phytopathol 2000, 90(8):801-811.

74. Zhang S, Yang X, Sun M, Sun F, Deng S, Dong H: Riboflavin-induced priming for pathogen defense in Arabidopsis thaliana. J Integr Plant Biol 2009, 51:167-174.

doi:10.1186/1471-2164-14-728

Cite this article as: Kugler et al:: Quantitative trait loci-dependent analysis of a gene co-expression network associated with Fusarium head blight resistance in bread wheat (Triticum aestivum L.). BMC Genomics 2013 14:728.

\section{Submit your next manuscript to BioMed Central and take full advantage of:}

- Convenient online submission

- Thorough peer review

- No space constraints or color figure charges

- Immediate publication on acceptance

- Inclusion in PubMed, CAS, Scopus and Google Scholar

- Research which is freely available for redistribution 